\title{
Elementos estructurales de la red social, fuentes de apoyo funcional, reciprocidad, apoyo comunitario y depresión en personas mayores en Chile
}

\author{
Lorena P. Gallardo-Peralta ${ }^{1 *}$, Esteban Sánchez-Moreno ${ }^{2}$, Andrés Arias-Astray ${ }^{3}$ y Ana Barrón López-de-Roda ${ }^{4}$ \\ 1 Escuela de Trabajo Social. Facultad de Ciencias Sociales y Jurídicas. Universidad de Taparacá (Arica, Chile). \\ 2 Departamento de Sociologia V. Facultad de Trabajo Social. Universidad Complutense de Madrid (España). \\ 3 Departamento de Trabajo Social y Servicios Sociales. Facultad de Trabajo Social. Universidad Complutense de Madrid (España). \\ 4 Departamento de Psicología Social. Facultad de Psicología. Universidad Complutense de Madrid (España).
}

\begin{abstract}
Resumen: La asociación entre apoyo social y depresión en el caso de las personas mayores constituye un objeto de estudio de especial relevancia en sociedades con altas tasas de envejecimiento, como es el caso de Chile. La presente investigación se centra en el papel jugado por variables de carácter psicosociológico: tipo de apoyo en función de la fuente de procedencia, reciprocidad en el apoyo según tipo de apoyo y fuente de procedencia, y apoyo comunitario. Se aplicaron escalas de depresión y apoyo social ampliamente utilizadas en una muestra $(n=493)$ de la región de Arica-Parinacota, en el Norte de Chile. Se realiza un análisis de regresión jerárquica y de ecuaciones estructurales. Los resultados sugieren que el cónyuge y los hijos son las fuentes de apoyo relevantes para los síntomas de depresión, sobre todo en sus funciones instrumental y emocional. La reciprocidad en el apoyo es una variable fundamental para dar cuenta de la relación existente entre fuentes de apoyo social y depresión.
\end{abstract}

Palabras clave: apoyo social; depresión; reciprocidad; personas mayores.

\section{Antecedentes}

Chile experimenta un acelerado proceso de envejecimiento de la población. En la actualidad el 13\% de su población está constituido por personas mayores y se estima que para el año 2025 esta cifra aumente al 19\%. En este contexto, distintos estudios destacan la alta prevalencia e incidencia de la depresión en el caso de las personas mayores (von Mühlenbrock et al., 2011; Luppa, Luck, Köning, Angermeyer y Riedel- Heller, 2012; Schwarzbach, Luppa, Forstmeier, König y Riedel-Heller, 2014; Vera, Buela y Zych, 2010). De ahí la relevancia que tiene analizar los factores que puedan actuar como mecanismos protectores frente a los síntomas depresivos en dicho grupo de edad.

La presente investigación explora la asociación entre depresión y apoyo social en la vejez. Diversos estudios confirman la relación de protección que el establecimiento de vínculos sociales durante la vejez supone frente la depresión (Altintas, Gallouj y Guerrien, 2012; Antonucci, Lansford y Akiyama, 2001; Feng, Ji y Xu, 2014; Li, Morrow-Howell, Proctor y Rubin, 2013; Vanderhorst y MacLaren, 2005). En términos generales, nuestro estudio se inscribe en el debate clásico sobre las relaciones entre elementos estructurales y funcionales del apoyo (Cohen y Wills, 1985) y su asociación con el desarrollo de síntomas de depresión. De los elementos estructurales considerados en la investigación previa en el caso de las personas mayores, es la frecuencia de contac-

* Dirección para correspondencia [Correspondence address]:

Lorena P. Gallardo Peralta. Departamento de Filosofía y Psicología. Facultad de Ciencias Sociales y Jurídicas. Universidad de Taparacá (Arica, Chile). 18 de Septiembre, 2222. Arica (Chile).

E-mail: lgallardo@uta.cl
Title: Structural dimension of social networks, sources of functional support, reciprocity, community support, and depression among elder people in Chile.

Abstract: The relation among aeging, social support, and depression is a key subject in societies with rising aeging rates, as Chile is. The present piece of research focus on the rol played by psychosociological variables: type of support by source, reciprocity in support by type and source of support, and communitary support. Validated and widely used scales for depression and social support where applied in a sample $(n=493)$ obtained from Arica-Parinacota region, north of Chile. Hierarchichal regression and structural equation model (SEM) are used. Results suggest that partner and adult children constitute the main sources of support (specially emotional and instrumental) against depression. Reciprocity is a key variable to account for the relation between sources of support and depression. Keywords: social support; depression; reciprocity; elderly.

tos en el seno de la red el factor que destaca en mayor medida por su relación con la salud mental y el deterioro psicológico. Se trata de una asociación que aparece en las investigaciones pioneras de los años 80, como por ejemplo la de Antonucci (1981), en la cual una mayor frecuencia de contactos se asoció con mayores puntuaciones en bienestar, felicidad y satisfacción vital. La investigación posterior sugiere que el impacto de la frecuencia de contactos sobre el deterioro psicológico está mediado por la evaluación que las personas mayores realizan de dicha interacción y por los niveles de satisfacción con los vínculos establecidos (Antonucci y Akiyama, 1987; Chao, 2011; Cruza-Guet, 2008). A la inversa, una escasa valoración de las relaciones sociales puede asociarse con indicadores negativos de salud y dar lugar a conductas de riesgo en la vejez (Moos, Brennan, Schutte y Moos, 2010). Para el caso específico de Chile, el estudio realizado por Herrera, Barros y Fernández (2011) pone de manifiesto que la valoración de las relaciones sociales incide positivamente en la calidad de vida de las personas mayores.

Usando un argumento más amplio, sería la utilidad emergente de las relaciones sociales el factor que en mayor medida determinaría el efecto de dichas relaciones durante el envejecimiento. Los antecedentes empíricos ponen de manifiesto la gran importancia de los aspectos funcionales del apoyo como elementos protectores frente al deterioro psicológico en general, y a la depresión en particular, en el caso de las personas mayores. En esta línea, ciertas investigaciones sugieren que los efectos sobre la depresión de los aspectos funcionales predominarían sobre los producidos por los elementos estructurales (Antonucci, Fuhrer y Dartigues, 1997; McLaughlin et al., 2012; Murrell, Norris y Chipley, 1992; Robitaille, Orpana y McIntosh, 2012). 
En todo caso, conviene matizar un debate susceptible de presentarse en términos de todo o nada, de interacción frente a satisfacción, de estructura frente a función. Para ello, es de gran utilidad introducir variables que incorporen en su propia definición la interacción entre estructura de la red social y el apoyo generado en la misma. Este sería un nivel de análisis plenamente psicosociológico, que en el caso de la presente investigación se concreta en la consideración de tres dimensiones de las redes de apoyo: la importancia de las fuentes en función del tipo de apoyo considerado, la reciprocidad en el apoyo funcional y el apoyo comunitario.

$\mathrm{El}$ debate sobre las fuentes del apoyo se relaciona directamente con la composición de las redes sociales de las personas mayores. Redes compuestas no solo por familiares, sino también por amigos, actuarían como amortiguador ante síntomas depresivos (Dean, Kolody y Wood, 1990; Litwin, 2011; Okabayashi, Liang, Krause, Akiyama y Sugisawa; 2004). Más aún, diversas investigaciones enfatizan la relevancia de una red informal heterogénea, es decir, una red social que no esté centrada en la familia (Chou y Chi, 2003; Fiori, Antonucci y Cortina, 2006). Así, la efectividad de las redes sociales en la generación de apoyo será diferente en función de las fuentes disponibles, de manera que los distintos tipos de apoyo (emocional, material, instrumental, informacional) mostrarán una relación diferente con la depresión en función de la fuente de dicho apoyo. Parece conveniente dirimir cómo la estructura de la red (fuentes disponibles) y las funciones del apoyo interactúan para generar resultados diferentes en las medidas de depresión en las personas mayores. Esta argumentación estaría en sintonía con las hipótesis clásicas de la especificidad del apoyo (Cohen y McKay, 1984; Cutrona, Russel y Rose, 1986), orientándose hacia uno de los debates recientes más interesantes en el estudio del apoyo social (Thoits, 1995).

En esta línea, conviene enfatizar la importancia que la reciprocidad en el apoyo social puede tener para las personas mayores. La investigación previa muestra resultados diversos. Autores como Kahn y Antonucci (1984) hacen referencia al concepto de "asimetría del apoyo", señalando que las personas mayores tienden a recibir más apoyo social del que brindan. En un sentido opuesto, Chappell y Funk (2011) muestran que las personas mayores son importantes proveedoras de apoyo social para sus familiares y amistades. Existen antecedentes, en todo caso, que ponen de manifiesto que a medida que se envejece disminuye la capacidad de dar apoyo, pero aumenta el apoyo social percibido por las personas mayores (Beel-Bates, Ingersoll-Dayton y Nelson, 2007; Shaw, Krause, Liang y Bennett, 2007). Parece necesario tomar en cuenta este debate, contemplando la reciprocidad en el apoyo en función del tipo de apoyo y de la fuente y destino del mismo en la relación recíproca. De esta manera, la variable "reciprocidad en el apoyo" se ocupa de la interacción entre elementos propios de la estructura de la red de apoyo y de los elementos funcionales del mismo con el objetivo de determinar qué tipo de relaciones recíprocas tienen una incidencia positiva en la salud mental de las personas mayores (Liang, Krause y Bennett, 2001; von dem Knesebeck, et al., 2009).

Otra de las dimensiones contempladas en el presente estudio es el apoyo comunitario, dada su importancia en la descripción y comprensión de las relaciones sociales (Lin, 1986). A través de esta variable se hace referencia al papel jugado por los barrios, las organizaciones de carácter voluntario (organizaciones religiosas, grupos de ocio, culturales, deportivos, asociaciones cívicas), la escuela y otros entornos sociales en los cuales los individuos se insertan durante una parte significativa de su tiempo (Herrero y Gracia, 2007). Tal y como señalan Gracia y Herrero (2006: 329), el análisis del apoyo social comunitario "incorpora tanto elementos objetivos (participación) como elementos subjetivos (sentimientos de integración y la percepción de estas organizaciones como un recurso al que acudir) y recoge, por tanto, los dos elementos de la distinción general entre integración social-estructural e integración sociopsicológica o emocional". De esta manera, el debate entre componentes estructurales (por ejemplo, participación en organizaciones comunitarias) y funcionales del apoyo (por ejemplo, sentido de pertenencia a la comunidad) tiene su reflejo en la dimensión comunitaria de las relaciones sociales (Harpham, Grant y Thomas, 2002). En este sentido, diversos autores afirman que las dificultades en la participación e integración comunitarias se relacionan con menores niveles de autoestima y mayor probabilidad de depresión y ansiedad (Bukov, Maas y Lampert, 2002; Gracia y Musitu, 1990). Igualmente, un sentido de integración y de participación comunitaria se asocia con la salud mental entre las personas mayores (Gracia, Herrero y Musitu, 2002; Mackean y Abbott-Chapman, 2012; Park, Jang, Lee, Ko y Chiriboga, 2014).

En este contexto, la bibliografía disponible pone de manifiesto la especial relevancia de la dimensión religiosa de la comunidad durante la vejez. La participación en actividades religiosas incluye un amplio número de situaciones que ponen en contacto al individuo con su comunidad. Estas actividades hacen referencia aquí tanto a la participación en los ritos religiosos (por ejemplo, la asistencia regular a ceremonias de culto y participación en grupos propios de la organización parroquial), como a la implicación en actividades de voluntariado basadas en la pertenencia y en el marco de las instituciones religiosas. La investigación previa ha puesto de manifiesto que la participación religiosa se relaciona con la depresión, de manera que la personas mayores implicadas en sus comunidades religiosas tienen una menor probabilidad de depresión, mientras que aquellas personas que carecen de filiación religiosa muestran comparativamente una mayor probabilidad de experimentar síntomas depresivos (Alvarado, Temper, Bresler y Thomas-Dobson, 1995; George, Kinghorn, Koenig, Gammon y Blazer, 2013; Sternthal, Williams, Musick y Buck, 2010; Yoon, 2008). Estos resultados señalan, por tanto, la centralidad de la dimensión religiosa de la comunidad en la vida de las personas mayores, sugiriendo que la implicación en este tipo de actividades actúa como un factor protector ante el deterioro psicológico. 
En resumen, el objetivo de este estudio consiste en analizar la relación entre diversas dimensiones del apoyo social y síntomas depresivos en personas mayores chilenas. De esta manera, se pretende ofrecer un modelo general basado en la noción de apoyo social que tome en cuenta los distintos procesos implicados en dicho concepto. En el marco de dicho modelo general, se pretende (1) analizar la relación entre elementos estructurales y funcionales del apoyo y las puntuaciones en depresión, (2) considerar la asociación entre apoyo social funcional y depresión tomando en consideración las distintas fuentes de procedencia (cónyuge, hijos/as, resto de familiares y amigos) y las funciones (emocional, consejo e instrumental) específicas del apoyo, (3) analizar el papel de las relaciones recíprocas de apoyo entre las personas mayores y las distintas fuentes de apoyo presentes en su red social y su relación con la depresión y (4) evaluar el papel del apoyo comunitario y su asociación con la depresión en el caso de las personas mayores.

\section{Método}

\section{Participantes}

Dados los objetivos del estudio, se planteó una investigación de tipo transversal. La muestra está conformada por 493 personas mayores chilenas que residen en el extremo norte de Chile (región de Arica y Parinacota). Se trata de una muestra con un margen de error (bajo el supuesto de muestreo aleatorio) del 4.4\% para un nivel de confianza del 95\%. En cuanto a sus características fundamentales, como se puede apreciar en la Tabla 1, el 86\% de las personas incluidas en la muestra tenía entre 60 y 79 años de edad (edad media de 71.47 años). El $67 \%$ eran mujeres y el $51 \%$ tenían estudios básicos. En cuanto al estado civil, la mayoría de la muestra tenía pareja (54\%), ya sea en matrimonio o en convivencia. De aquellos que no cuentan con pareja, la mayoría de éstos eran viudos/as (25\% de la muestra). Se trata de una muestra que incluye tanto personas residentes en zonas urbanas $(86 \%)$ como en zonas rurales de valles pre-cordilleranos y en zonas altiplánicas de los Andes. Finalmente, el 30\% pertenecía a una etnia originaria chilena, siendo la aymara la más frecuente ( $85 \%$ de los casos). Todas estas características constituyen un reflejo fiel de la composición demográfica y social de las personas mayores de 65 años en la región de Arica-Parinacota.

\section{Procedimiento}

La aplicación del cuestionario se realizó a través de entrevista personal. Cuatro graduados en trabajo social realizaron el trabajo de campo entre los meses de abril y mayo de 2011. Para realizar el estudio se siguieron dos estrategias. Por un lado, para la aplicación en la zona urbana de Arica se estableció contacto con la institución gubernamental de personas mayores (Servicio Nacional de Adultos Mayores), que facilitó la aplicación de los cuestionarios y aportó informa- ción de las principales agrupaciones de personas mayores en la ciudad. Por otro lado, la aplicación del cuestionario en las zonas rurales/altiplánicas supuso afrontar grandes dificultades, como el difícil acceso a los poblados (por no contar con carreteras asfaltadas) o la existencia de horarios restringidos de luz pública y en los hogares (por tanto con horarios restringidos de aplicación). En el caso específico de localidades altiplánicas se añaden las dificultades que entrevistador pueda presentar en términos físicos (por la altitud geográfica) y los rasgos culturales centrados en la desconfianza al extraño, siendo imprescindible contar con el apoyo de un agente social de la zona. Por todos esos motivos, en las zona rurales (Valle de Codpa) y altiplánicas (Putre y Socoroma) se contactaron agentes sociales clave. En el Valle de Codpa se contactó con el párroco del poblado y la presidenta del Club de Adultos Mayores. El cuestionario se aplicó en las dependencias de la Junta de Vecinos y asimismo se realizó un puerta a puerta por el pueblo. Procedimiento similar se realizó en las zonas altiplánicas de Putre y Socoroma, en las que se localizó al párroco de Putre y a dirigentes de agrupaciones de personas mayores, aplicándose los cuestionarios en una reunión convocada por los dirigentes en cada localidad. Aunque laborioso y costoso, este procedimiento de aplicación de cuestionarios en la zonas rurales y altiplánicas permitió obtener una muestra representativa como resultado final del proceso.

Tabla 1. Características de la muestra.

\begin{tabular}{llc}
\hline Variable & Categorías & $\begin{array}{c}\text { Frecuencia } \\
n(\%)\end{array}$ \\
\hline Edad & $60-69$ años & $207(42 \%)$ \\
(por grupos) & $70-79$ años & $216(44 \%)$ \\
& $80-89$ años & $63(13 \%)$ \\
& 90 y más años & $7(1 \%)$ \\
\hline Sexo & Mujer & $330(67 \%)$ \\
& Hombre & $163(33 \%)$ \\
\hline Escolaridad & Sin studios & $48(10 \%)$ \\
& Educación básica & $251(51 \%)$ \\
& Educación media & $128(26 \%)$ \\
& Educación superior & $66(13 \%)$ \\
\hline Estado conyugal & Casado/a & $243(49 \%)$ \\
& Coviviente & $22(5 \%)$ \\
& Soltero/a & $52(11 \%)$ \\
& Viudo/a & $123(25 \%)$ \\
& Divorciado/a o separado/a de hecho & $53(10 \%)$ \\
\hline Localidad en la & Urbana (ciudad de Arica) & $425(86 \%)$ \\
que reside & Rural (Codpa y poblados cercanos) & $17(3 \%)$ \\
& Altiplánico (Putre, Socoroma u otro) & $51(11 \%)$ \\
\hline Pertenece a etnia & Sí pertenece & $147(30 \%)$ \\
originaria chilena & No pertenece & $346(70 \%)$ \\
\hline
\end{tabular}

\section{Instrumentos}

Inventario de Recursos Sociales en Personas Mayores de Díaz Veiga (1987; véase Fernández Ballesteros, 2000). Esta escala explora aspectos estructurales de las redes sociales (tamaño y frecuencia de contactos), funcionales (apoyo 
emocional, instrumental e informacional) y la satisfacción con las relaciones. En la presente investigación se consideraron diversas fuentes informales de apoyo tales como: cónyuge, hijos, familiares y amigos. En concreto, este cuestionario se utilizó para operacionalizar las variables estructurales del apoyo (tamaño de la red y frecuencia de contactos) y la satisfacción con las relaciones dentro de la red. El índice de consistencia interna (alpha de Cronbach) fue de .81.

Cuestionario de Apoyo Social Percibido de Gracia et al. (2002). Cuestionario que evalúa las dimensiones de apoyo emocional, consejo y ayuda y la reciprocidad en el apoyo. Ofrece también una puntuación total para el apoyo funcional y la reciprocidad en el apoyo, así como el número de los componentes de la red del apoyo. Este instrumento permite además obtener puntuaciones separadas para las distintas fuentes de apoyo social: familiares, vecinos, hermanos, hijos/as. El índice de consistencia interna obtenido (alpha de Cronbach) fue de .93 .

Apoyo Comunitario. Se utilizaron dos indicadores de apoyo social comunitario, a saber, la participación en organizaciones de carácter religioso y la participación en las actividades de los denominados clubes de adultos mayores. Ambas instancias captan adecuadamente la importancia de la comunidad como fuente de apoyo social y constituyen dos de las formas más importantes de participación comunitaria de las personas mayores en Chile en general, y en la región de Arica - Parinacota en particular. Una medida de ítem único para la participación religiosa preguntaba la frecuencia con la cual la persona entrevistada acudía a la iglesia o a reuniones religiosas, midiendo dicha frecuencia en una escala de cinco puntos $(0=$ nunca o casi nunca; $4=$ más de una vez a la semana). Para medir la participación en clubes de adultos mayores se preguntó a las personas en el estudio la frecuencia con la cual acudían a dichas organizaciones, registrando la asistencia en la misma escala citada. Se trata, por tanto, de dos variables construidas a partir de un único indicador para cada una.

Escala Geriátrica de Depresión de Brink et al. (1982). La versión original consta de 30 preguntas, si bien en este estudio se utilizó la versión abreviada, que incluye solo 15. Esta última conserva la efectividad de la escala original, mejorando la facilidad de administración. Según FernándezBallesteros (2000, p.395) puntuaciones entre 6-9 indican depresión leve y puntuaciones a partir de 10 indican depresión establecida. El índice de consistencia interna (alpha de Cronbach) fue de .77 . Es un instrumento ampliamente utilizado para evaluar la depresión en personas mayores, y su versión en castellano (Baker y Espino, 1997) ha sido utilizada exitosamente en población chilena por Hoyl, Valenzuela y Marín (2000).

\section{Modelos de Análisis}

El análisis de datos se realizó en tres fases. En un primer momento se realizaron los análisis descriptivos de las principales variables del estudio. En segundo lugar, se realizó un análisis de regresión jerárquica que supuso la formulación de 5 modelos con niveles de complejidad creciente en la incorporación de variables, a través de la versión 19 del programa SPSS. Se parte de un modelo limitado a los aspectos estructurales (modelo 1) que incluye la satisfacción con los mismos. Los tres modelos siguientes $(2,3$ y 4$)$ incorporan de manera progresiva las fuentes de apoyo, comenzando por las más relevantes desde el punto de vista teórico (cónyuge e hijos) y finalizando por la reciprocidad con respecto a las distintas fuentes, en consonancia con nuestro análisis de los antecedentes. El modelo 5 constituiría un modelo general de apoyo social, al añadir a las variables anteriores el apoyo social comunitario. Por último, se formuló un modelo general de relación entre las variables, que fue puesto a prueba a través de un análisis de ecuaciones estructurales con el programa AMOS versión 19. En este último caso, para estimar el ajuste de los datos al modelo se utilizaron los siguientes índices: $\chi^{2}$, RMSEA (Root Mean Error of Approximation), CFI (Comparative Fit Index), AGFI (Adjusted Goodness-of-fit Index) y NNFI (Non-normed Fit Index). Valores por debajo de .05 para RMSEA y por encima de .95 para el resto de índices citados sugieren un buen ajuste.

\section{Resultados}

La Tabla 2 resume los estadísticos descriptivos de las variables generales del estudio. Destaca que la media de la muestra en la medida de depresión (4.24) se traduce en que un $23 \%$ de la muestra se encontraría en la categoría de depresión leve y un $6 \%$ en la de depresión establecida, según el estándar de clasificación del cuestionario utilizado. Además, la media de la muestra en los indicadores de apoyo social, tanto estructurales como funcionales, se sitúa en un punto relativamente bajo del rango posible. No obstante, destacan los altos niveles de satisfacción con las relaciones sociales y el alto grado relativo de reciprocidad en todas las dimensiones del apoyo.

Los resultados obtenidos a través de los modelos de regresión jerárquica se ofrecen en la tabla 3. Si bien todos modelos son estadísticamente significativos, solo los modelos 3 y 4 suponen un incremento significativo en su capacidad explicativa de las puntuaciones en depresión con respecto al modelo 1, que incorpora únicamente los aspectos estructurales. Ambos son modelos de redes sociales por fuentes, ya que consideran tanto los aspectos estructurales como las funciones del apoyo en función de las fuentes de dicho apoyo (modelo 3), destacando la importancia de la reciprocidad en el mismo (modelo 4). 
Tabla 2. Estadísticos descriptivos de las principales variables del estudio.

\begin{tabular}{|c|c|c|c|}
\hline & $M$ & DT & Rango observado \\
\hline Depresión & 4.24 & 3.14 & $0-15$ \\
\hline Tamaño de la red & 2.09 & 1.22 & $0-18$ \\
\hline Frecuencia de contactos & 12.19 & 3.26 & $4-18$ \\
\hline Satisfacción con relaciones & 13.43 & 2.72 & $4-18$ \\
\hline Participación religiosa & 2.28 & 1.01 & $0-4$ \\
\hline Participación clubes adultos mayores & 1.17 & .40 & $0-4$ \\
\hline Cónyuge apoyo emocional & 4.07 & 3.44 & $0-10$ \\
\hline Cónyuge apoyo informacional (consejo) & 4.05 & 3.43 & $0-10$ \\
\hline Cónyuge apoyo instrumental & 4.12 & 3.48 & $0-10$ \\
\hline Cónyuge reciprocidad emocional & 2.06 & 1.24 & $0-5$ \\
\hline Cónyuge reciprocidad informacional & 2.04 & 1.23 & $0-5$ \\
\hline Cónyuge reciprocidad instrumental & 2.05 & 1.24 & $0-5$ \\
\hline Hijos apoyo emocional & 7.87 & 7.36 & $0-50$ \\
\hline Hijos apoyo informacional (consejo) & 7.70 & 7.04 & $0-50$ \\
\hline Hijos apoyo instrumental & 7.82 & 7.30 & $0-50$ \\
\hline Hijos reciprocidad emocional & 3.99 & 3.29 & $0-25$ \\
\hline Hijos reciprocidad informacional & 3.94 & 3.29 & $0-25$ \\
\hline Hijos reciprocidad instrumental & 4.01 & 3.33 & $0-25$ \\
\hline Familiares apoyo emocional & 3.44 & 5.54 & $0-36$ \\
\hline Familiares apoyo informacional (consejo) & 3.23 & 5.07 & $0-31$ \\
\hline Familiares apoyo instrumental & 3.34 & 5.24 & $0-32$ \\
\hline Familiares reciprocidad emocional & 1.73 & 2.31 & $0-20$ \\
\hline Familiares reciprocidad informacional & 1.67 & 2.15 & $0-16$ \\
\hline Familiares reciprocidad instrumental & 1.69 & 2.19 & $0-16$ \\
\hline Amigos apoyo emocional & 1.39 & 2.88 & $0-20$ \\
\hline Amigos apoyo informacional (consejo) & 1.27 & 2.66 & $0-24$ \\
\hline Amigos apoyo instrumental & 1.35 & 2.87 & $0-22$ \\
\hline Amigos reciprocidad emocional & .70 & .98 & $0-12$ \\
\hline Amigos reciprocidad informacional & .67 & .89 & $0-12$ \\
\hline Amigos reciprocidad instrumental & .68 & .93 & $0-12$ \\
\hline Apoyo emocional total & 16.76 & 9.67 & $0-60$ \\
\hline Apoyo informacional total (consejo) & 16.24 & 9.31 & $0-60$ \\
\hline Apoyo instrumental total & 16.94 & 9.30 & $0-60$ \\
\hline Reciprocidad emocional total & 8.48 & 5.04 & $0-30$ \\
\hline Reciprocidad informacional total & 8.32 & 4.93 & $0-30$ \\
\hline Reciprocidad instrumental total & 8.42 & 4.96 & $0-30$ \\
\hline
\end{tabular}

Considerando el modelo 4 ("redes sociales por fuentes"), los resultados muestran la importancia de los elementos estructurales, $y$ en concreto de la frecuencia de contacto $(\beta=$ $.188 ; p<.01)$ y del tamaño de la red significativa $(\beta=-.239$; $p<.05)$. No se encontraron asociaciones significativas en el caso de la satisfacción con las relaciones. Los resultados también confirman la importancia de las dimensiones funcionales, señalando dos como las más relevantes, la instrumental y la emocional. En el caso del apoyo instrumental son dos las fuentes que se relacionan con la depresión, en ambos casos en un sentido negativo: el apoyo instrumental procedente del cónyuge $(\beta=-1.286 ; p<.05)$ y el apoyo instrumental de la familia extensa $(\beta=-.638 ; p<.01)$. Además, la reciprocidad en el apoyo emocional del cónyuge se relaciona con mayores niveles de depresión $(\beta=2.033 ; p<.01)$ y con menores niveles en el caso de los hijos $(\beta=-1.239 ; p<$ $.001)$.

El modelo 5 (apoyo social comunitario) no supuso un cambio significativo en el estadístico de contraste, motivo por el cual no se muestra en la Tabla 3.
A partir de estos resultados, y atendiendo a los antecedentes teóricos y empíricos disponibles, se formuló un modelo para contrastar con los datos a través de un análisis de ecuaciones estructurales. Este modelo hipotetizó que las puntuaciones en la variable depresión estarían directamente relacionadas con la frecuencia de contactos dentro de la red, con el apoyo instrumental de los hijos y de la familia extensa (en ambos casos en un sentido estadísticamente negativo), con la reciprocidad en el apoyo emocional del cónyuge (asociación positiva) y de los hijos (asociación negativa) y con la participación en organizaciones religiosas (asociación negativa) como medida de apoyo comunitario. Además, el tamaño de la red tendría un efecto indirecto en los niveles de depresión a través de su relación con la frecuencia de contactos y con las dimensiones funcionales del apoyo anteriormente mencionadas. Para todas las variables mencionadas, los indicadores de asimetría y curtosis se encontraban por debajo de los límites recomendados por Kline (2005). No obstante, el coeficiente de normalidad multivariada de Mardia (1970) fue de 80.927, sugiriendo la ausencia de normalidad al considerar en conjunto todas las variables del modelo. Por ese motivo, 
se utilizaron estimadores de máxima verosimilitud complementando el análisis con la técnica de muestreo "bootstrap- ping" y el método de estimación de intervalos de confianza no sesgado.

Tabla 3. Regresión jerárquica para la variable depresión según modelos teóricos.

\begin{tabular}{|c|c|c|c|c|}
\hline \multicolumn{2}{|l|}{$\begin{array}{l}\text { MODELO } \\
\left(r^{2}\right)(\text { cambio en } F)\end{array}$} & $B$ & E.T. & $\beta$ \\
\hline Modelo 1. & Frecuencia del contacto & .151 & .065 & $.156^{*}$ \\
\hline Estructura y satisfacción. & Satisfacción con las relaciones sociales & -.201 & .077 & $-.175^{* *}$ \\
\hline$(.010)(2.503)$ & Tamaño de la Red Significativa & -.085 & .121 & -.033 \\
\hline Modelo 2. & Frecuencia del contacto & .156 & .066 & $.161 * *$ \\
\hline Redes Sociales por fuentes I & Satisfacción con las relaciones sociales & -.121 & .084 & -.105 \\
\hline \multirow[t]{7}{*}{$(.016)(1.445)$} & Tamaño de la Red Significativa & -.017 & .147 & -.007 \\
\hline & Apoyo emocional del cónyuge & -.239 & .400 & -.338 \\
\hline & Consejo del cónyuge & .177 & .379 & .249 \\
\hline & Apoyo Instrumental del cónyuge & -.038 & .282 & -.054 \\
\hline & Apoyo emocional de los hijos & -.077 & 117 & -.203 \\
\hline & Consejo de los hijos & -.043 & .079 & -.108 \\
\hline & Apoyo Instrumental de los hijos & .106 & .092 & .280 \\
\hline Modelo 3. & Frecuencia del contacto & .169 & .065 & $.175^{* *}$ \\
\hline Redes Sociales por fuentes II & Satisfacción con las relaciones sociales & -.130 & .084 & -.113 \\
\hline \multirow[t]{13}{*}{$(.035)(2.474 *)$} & Tamaño de la Red Significativa & -.348 & .251 & -.134 \\
\hline & Apoyo emocional del cónyuge & -.270 & .398 & -.381 \\
\hline & Consejo del cónyuge & .250 & .377 & .353 \\
\hline & Apoyo Instrumental del cónyuge & -.126 & .281 & -.180 \\
\hline & Apoyo emocional de los hijos & -.137 & 118 & -.361 \\
\hline & Consejo de los hijos & -.074 & .079 & -.189 \\
\hline & Apoyo Instrumental de los hijos & .148 & .095 & .390 \\
\hline & Apoyo emocional de los familiares & .292 & .144 & $.582 *$ \\
\hline & Consejo de los familiares & -.037 & .122 & -.069 \\
\hline & Apoyo Instrumental de los familiares & -.360 & .134 & -.689 \\
\hline & Apoyo emocional de los amigos & -.340 & .215 & -.409 \\
\hline & Consejo de los amigos & .207 & .215 & .234 \\
\hline & Apoyo Instrumental de los amigos & .128 & .204 & .152 \\
\hline Modelo 4. & Frecuencia del contacto & .182 & .066 & $.188^{* *}$ \\
\hline Redes Sociales por fuentes III & Satisfacción con las relaciones sociales & -.109 & .085 & -.095 \\
\hline \multirow{25}{*}{$(.063)(2.079 * *)$} & Tamaño de la Red Significativa & -.619 & .270 & $-.239 *$ \\
\hline & Apoyo emocional del cónyuge & -.286 & .404 & -.404 \\
\hline & Consejo del cónyuge & .013 & .393 & .019 \\
\hline & Apoyo Instrumental del cónyuge & -.901 & .412 & $-1.286^{*}$ \\
\hline & Apoyo emocional de los hijos & -.059 & .131 & -.155 \\
\hline & Consejo de los hijos & -.020 & .090 & -.051 \\
\hline & Apoyo Instrumental de los hijos & .173 & 102 & .456 \\
\hline & Apoyo emocional de los familiares & .250 & 163 & .499 \\
\hline & Consejo de los familiares & -.010 & 130 & -.019 \\
\hline & Apoyo Instrumental de los familiares & -.333 & 139 & $-.638^{* *}$ \\
\hline & Apoyo emocional de los amigos & -.206 & .339 & -.247 \\
\hline & Consejo de los amigos & .233 & 235 & .262 \\
\hline & Apoyo Instrumental de los amigos & .585 & 349 & .696 \\
\hline & Reciprocidad en Apoyo Emocional del cónyuge & 2.850 & 1.166 & $2.033^{* *}$ \\
\hline & Reciprocidad en Consejo del cónyuge & .273 & .753 & .194 \\
\hline & Reciprocidad en Apoyo Instrumental del cónyuge & -1.135 & 1.000 & -.807 \\
\hline & Reciprocidad en Apoyo Emocional de los hijos & -.911 & .262 & $-1.239 * * *$ \\
\hline & Reciprocidad en Consejo de los hijos & .251 & .266 & .340 \\
\hline & Reciprocidad en Apoyo Instrumental de los hijos & .069 & .304 & .094 \\
\hline & Reciprocidad en Apoyo Emocional de los familiares & .438 & .342 & .443 \\
\hline & Reciprocidad en Consejo de los familiares & -.529 & .328 & -.504 \\
\hline & Reciprocidad en Apoyo Instrumental de los familiares & -.051 & .377 & -.050 \\
\hline & Reciprocidad en Apoyo Emocional de los amigos & -.741 & .793 & -.452 \\
\hline & Reciprocidad en Consejo de los amigos & -.004 & .725 & -.002 \\
\hline & Reciprocidad en Apoyo Instrumental de los amigos & -.532 & .858 & -.315 \\
\hline
\end{tabular}


El modelo descrito no alcanzó niveles de ajuste satisfactorio en ninguno de los estadísticos utilizados, procediéndose a su revisión a partir de criterios teóricos y con el apoyo de los índices de modificación. El modelo resultante aparece en la figura 1 (no se incluyen los términos de error para facilitar su lectura) y muestra un excelente ajuste a los datos $\left(\chi^{2}\right.$ $=13.68 ; p=.134)$, tal y como ponen de manifiesto los valores de los índices de bondad del ajuste (RMSEA $=.03$; CFI $=.99 ; \mathrm{AGFI}=.97$; NNFI $=.99)$. Los términos de error de las variables "reciprocidad emocional" y "apoyo instrumental" se encuentran correlacionados para ambas fuentes (cónyuge e hijos). Se trata de un modelo que incluye tanto di- mensiones estructurales como funcionales del apoyo. Ahora bien, mientras que los modelos de regresión señalaban el tamaño de la red como una variable fundamental, el análisis de ecuaciones estructurales sugiere que es una dimensión muy específica de dicha variable la que tiene un papel relevante en la depresión en el caso de los adultos mayores, a saber, el número de hijos vivos. De hecho, el ajuste del modelo supuso la eliminación del tamaño de la red en general por la variable "número de hijos vivos". La relación de esta variable con la depresión es indirecta, a través del apoyo funcional procedente, precisamente, de los hijos.

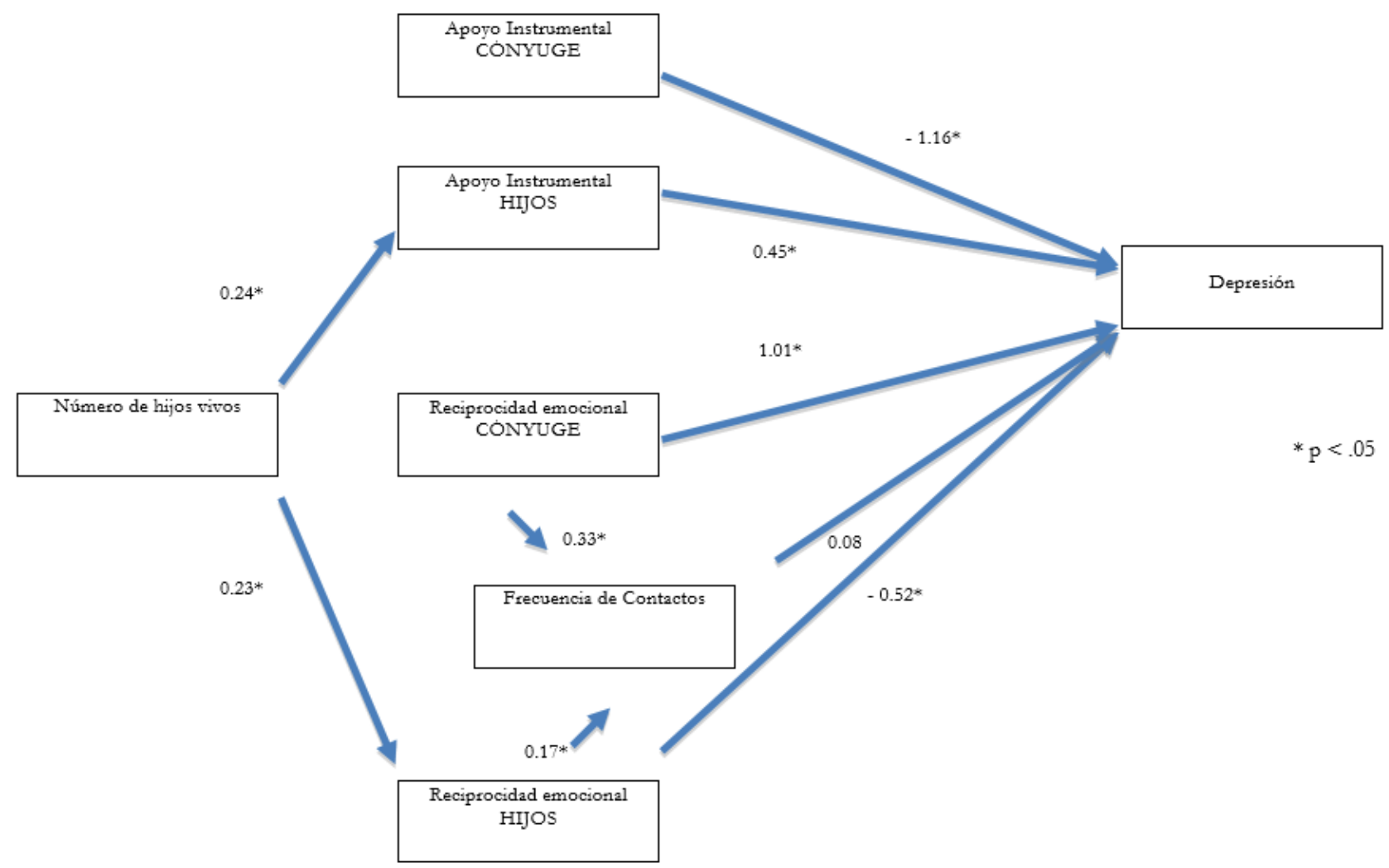

Figura 1. Modelo de ecuaciones estructurales $\left(\chi^{2}=13.68 ; p=.134\right.$; g.l. $\left.=9\right)$

Todo ello se debe al hecho de que las fuentes estadísticamente asociadas con la depresión se redujeron a dos en los resultados: cónyuge e hijos. En efecto, y a pesar de que en los modelos de regresión jerárquica aparecía una asociación significativa para el apoyo instrumental de los familiares, no fue posible ajustar un modelo de ecuaciones estructurales que contuviera dicha variable. Como puede apreciarse en la figura 1, es el apoyo instrumental de los hijos la variable que muestra una relación significativa. Llama la atención que en el caso del apoyo instrumental las dos fuentes que se relacionan con la depresión lo hagan en sentido contrapuesto: el apoyo instrumental del cónyuge se relaciona negativamente con la depresión $(\beta=-1.164 ; p<.05)$ y el apoyo instrumen- tal de los hijos se asocia con mayores puntuaciones en dicha variable $(\beta=.453 ; p<.05)$.

La segunda dimensión funcional del apoyo que aparece en el modelo recogida en la figura 1 es la emocional. En concreto, es la reciprocidad en el apoyo emocional el factor que muestra una asociación significativa con las puntuaciones en depresión. Además, esta asociación se da únicamente para dos fuentes, y nuevamente con un sentido inverso: la reciprocidad emocional se asocia positivamente con la variable depresión en el caso del cónyuge $(\beta=1.007 ; p<.05)$, mientras que la reciprocidad emocional con los hijos se asocia con menores puntuaciones en dicha variable $(\beta=-.520 ; p$ $<.01)$. Ambos procesos, a su vez, se relacionan con una ma- 
yor frecuencia de contactos dentro de la red de apoyo. La asociación de esta última variable con la depresión no alcanzó niveles estadísticamente significativos $(\beta=.08 ; p=.072)$, pero se mantuvo en el modelo porque, sin afectar al ajuste del modelo en su conjunto, tiene las notables implicaciones teóricas y empíricas que serán expuestas en la discusión.

\section{Discusión}

Los resultados expuestos en el presente artículo tienen implicaciones relevantes para el estudio de la relación entre el deterioro psicológico (en este caso, depresión) y las diversas dimensiones (estructurales y funcionales) del apoyo social. El modelo resultante y recogido en la figura 1 (al igual que las ecuaciones de regresión jerárquica) incluye elementos estructurales y funcionales. No obstante, parece adecuado señalar que son estos últimos los que desempeñan un papel de mayor relevancia en el caso de nuestra muestra de personas mayores en Chile. En primer lugar, los resultados sugieren que el tamaño de la red se relaciona con menores puntuaciones en la medida de depresión. Ahora bien nuestros análisis señalan que es una dimensión muy concreta del tamaño de la red la que es relevante (el número de hijos vivos), mostrando además una relación indirecta con el deterioro, precisamente a través del apoyo instrumental y emocional prestado por ellos. Esto sugiere que los elementos estructurales supondrían una condición de posibilidad, el contexto necesario del cual emergen las funciones propias de la interacción social (Thoits, 1983).

Un argumento similar se puede utilizar para dar cuenta de los resultados relativos a la frecuencia de contactos, otra de las variables estructurales clásicas. En los seis modelos de regresión recogidos en la tabla 3 existe una relación significativa con la depresión, sugiriendo que a mayor frecuencia del contacto, mayor es la depresión. Este resultado sería consistente con una hipótesis de movilización de la red de apoyo en situaciones de necesidad. Ahora bien, nuestro análisis de ecuaciones estructurales no ofrece un coeficiente estadísticamente significativo en la relación entre frecuencia de contactos y depresión. En dicho modelo, esta variable estructural estaría relacionada con la reciprocidad del apoyo, de manera que la existencia de dicha reciprocidad incrementa la frecuencia de contactos. Nuevamente nos encontramos con una dimensión estructural que constituiría el contexto en el cual se despliega la interacción social. Planteado en términos más amplios, podría afirmarse que la integración social es el contexto necesario para el apoyo social (Sánchez y Barrón, 2003).

Todo ello sugiere la necesidad de ir más allá del debate general y considerar elementos específicos que ayuden a dar cuenta de la complejidad de dicho debate. La contribución del presente artículo en dicho sentido se concreta en los resultados relativos a dos dimensiones del apoyo en las cuales no se establece una separación nítida entre funciones y estructura, sino que en gran medida están constituidas por la interacción sistemática entre ambos elementos. Se trata de la composición de la red y la eficacia diferencial de las fuentes de apoyo, por un lado, y de la reciprocidad en el apoyo, por otro.

Con respecto al primer elemento, los resultados obtenidos señalan que el cónyuge y los hijos constituyen las fuentes fundamentales de apoyo social (en concreto, instrumental y emocional) en el caso de las personas mayores. A pesar de que estos resultados son contradictorios con la evidencia empírica que señala que redes sociales heterogéneas se relacionan con menores niveles de deterioro, lo cierto es que son consistentes con investigaciones anteriores que sugieren que en el contexto chileno la familia (prácticamente de forma exclusiva) es la entidad encargada de proveer cuidado y ayuda material (servicios) y psicosocial para las personas mayores (Arechabala y Miranda, 2002; Barros y Muñoz, 2003; Herrera y Kornfeld, 2008, Herrera, Rojas, Campos y Fernández, 2013). En términos más generales, la evidencia empírica en personas mayores pone de manifiesto la relevancia de la figura del cónyuge y de los hijos. En el caso de las personas mayores casadas, se plantea que el cónyuge/pareja constituye la fuente primaria de compañía, de proximidad íntima y de bienestar (Peters, Hoyt, Babchuk, Kaiser e Iijima, 1987; Quinn, Hughston y Hunter, 1984). En esta línea Larson y Silverstein (2004) observaron que los adultos mayores sin hijos muestran menores niveles de apoyo informal. Cuando se da esta circunstancia los apoyos formales (por ejemplo, los servicios de ayuda a domicilio) no son efectivos para compensar dicha carencia. En cambio, cuando la ausencia de una fuente se refiere a otros familiares (como es el caso de los hermanos) o a las amistades, estas típicamente son reemplazadas en sus funciones de manera eficaz por el cónyuge y los hijos (Marcil- Gratton y Légaré, 1992).

En este contexto, la relación negativa entre apoyo instrumental del cónyuge y depresión es consistente con la bibliografía previa. De hecho, el apoyo instrumental del cónyuge puede generar un sentimiento de afrontamiento conjunto de las crisis propias de la vejez. Ahora bien, en el caso de los hijos nuestros resultados muestran que el apoyo instrumental procedente de dicha fuente se asocia con mayores puntuaciones en depresión. Una posible explicación es que el hecho de recibir apoyo material, ya sea por circunstancias económicas o bien de salud, puede generar sentimientos de vergüenza, incapacidad y/o dependencia. Se debe tener en cuenta que el apoyo social puede llegar a constituirse en un factor de estrés. Esta circunstancia es especialmente aplicable al caso específico de personas mayores, para las cuales recibir apoyo instrumental o material por parte de los hijos puede estar asociado a un sentimiento de dependencia (Chappell y Funk, 2011) y por lo tanto puede no ser funcional como factor protector en el caso del deterioro psicológico (Robitaille et al., 2012).

Además, esta explicación permite dar cuenta de la relación negativa existente entre la recriprocidad en el apoyo emocional con los hijos y la depresión. En este punto, el hecho de que la personas mayores puedan establecer una relación recíproca de apoyo con sus hijos contribuiría al desarrollo de 
sentimientos de autoeficacia, es decir, daría lugar a sentimientos positivos para prevenir los síntomas de depresión. Se trataría de actuaciones exitosas - en este caso el retribuir el apoyo - que generan una sensación de control y eficacia, sentimientos que a su vez incrementan la autoestima de las personas (Thoits, 2011) y previenen sentimientos de dependencia y aislamiento.

Más complicada es la explicación de la asociación positiva encontrada entre la reciprocidad en el apoyo emocional cuando la fuente es el cónyuge y las puntuaciones en depresión. No obstante, es posible que dicha reciprocidad no sea experimentada por las personas mayores que forman parte de la muestra como un intercambio voluntario, sino más bien como una imposición situacional que, en contextos de tensión, constituya una fuente de estrés añadida, una obligación que añade dificultades a las situaciones vitales que puedan experimentarse (Choi y McDougall, 2009; Koizumiy et al., 2005)

Llama la atención la ausencia de una asociación significativa entre las medidas de apoyo comunitario utilizadas y las puntuaciones en depresión. Existen diversas explicaciones potenciales para dicho resultado, que difiere de la evidencia empírica acumulada, tal y como se ha analizado anteriormente. Es posible que los indicadores utilizados para medir el apoyo comunitario no sean adecuados para tal fin. En contraste con las medidas utilizadas para el resto de variables consideradas en el estudio, se trata de indicadores únicos (nivel de participación en clubes de adultos mayores y participación en organizaciones religiosas) que miden el apoyo comunitario desde una perspectiva estructural, en contraste con estudios previos que operacionalizan dicho concepto a través del sentido de pertenencia a la comunidad (Fowler, Wareham-Fowler y Barnes, 2013; Herrero y Gracia, 2007; Mair, Diez-Roux y Morenoff, 2010). Ahora bien, la evidencia empírica en este sentido no es concluyente para el caso específico de la asociación entre participación religiosa y depresión. Es cierto que determinados estudios que utilizan una variable de indicador único para medir este tipo de participación no encuentran asociación con el bienestar (González-Celis y Gómez-Benito, 2013). Sin embargo, son abundantes los estudios que, utilizando una medida muy similar a la descrita para la presente investigación, encuentran una asociación significativa entre participación religiosa y depresión (Park et al., 2014; Rushing, Corsentino, Hames, SachsEricsson y Steffens, 2013), incluyendo estudios longitudinales (Barton, Miller, Wickramaratne, Gameroff y Weissman, 2013).

Así, es posible que no sea la forma concreta de operacionalización (indicador único) la que explique por completo nuestros resultados en torno al apoyo comunitario, sino más bien la dimensión a la cual atienden. Dicho de otra forma, es posible que la ausencia de una asociación entre participación comunitaria y depresión se deba a que los dos indicadores utilizados hacen referencia al apoyo comunitario desde una perspectiva estructural, pero no sean adecuados para captar sus funciones. Estudios previos sugieren que, al igual que en el caso del apoyo social son las funciones (instrumental, emocional, consejo, material) del mismo el elemento que da cuenta del impacto de las relaciones sociales en la salud mental, también el apoyo comunitario será especialmente significativo para el bienestar de las personas a través de dichas funciones (Gracia y Herrero, 2006). En esta línea, la investigación de Roh, Lee y Yoon (2013) analiza el papel jugado por la religiosidad en una muestra de personas inmigrantes de origen asiático en Estados Unidos. Su medida de religiosidad incluía diversas subescalas que, medidas a través de varios ítems, permiten operacionalizar cuatro dimensiones: valores y creencias religiosas; práctica religiosa privada; afrontamiento religioso y; apoyo social (funcional) de origen religioso. De todas estas subescala, solo la última mostró una asociación con medidas de bienestar general, ansiedad y depresión entre las personas mayores que formaban la muestra. De esta manera, el apoyo funcional generado en el ámbito religioso parece constituir el elemento protector fundamental de la práctica religiosa en el caso de las personas mayores.

Las medidas de apoyo comunitario utilizadas en nuestra investigación no captan dicha dimensión funcional del apoyo, lo que puede explicar la falta de asociación con las puntuaciones en depresión. Más aún teniendo en cuenta que nuestros resultados señalan, precisamente, la gran importancia de los elementos funcionales del apoyo, si se compara con sus elementos estructurales. Esta limitación de nuestro estudio invita al desarrollo de una línea de investigación posterior que incorpore medidas adecuadas para captar la dimensión funcional del apoyo comunitario. Estas investigaciones deberían incluir igualmente medidas funcionales del resto de fuentes potenciales de apoyo, con el objetivo de discernir la medida en la cual la falta de asociación entre apoyo comunitario y depresión que sugiere nuestro estudio se debe a problemas de medición y/o a la gran importancia relativa que nuestros resultados sugieren para las fuentes de apoyo familiar más cercanas e íntimas (cónyuge e hijos).

Una segunda limitación tiene que ver con el diseño de investigación, ya que los resultados obtenidos derivan de datos de carácter transversal. Esta circunstancia ha de ser destacada e invita a proceder con cautela en la interpretación de la dirección causal de las asociaciones halladas. Los modelos teóricos formulados y los análisis de datos realizados contemplan las puntuaciones en la variable depresión en tanto que influidas por las diversas dimensiones del apoyo social consideradas. Esta decisión se deriva del examen realizado de los antecedentes teóricos y empíricos. Sin embargo, diversos autores han señalado la existencia de un patrón de asociación complejo entre el apoyo social y la salud mental, que incluiría el análisis de los determinantes del apoyo social (Barrera, 2000). En términos más concretos, y en el contexto de nuestra investigación, es de especial relevancia la bibliografía previa que pone de manifiesto la influencia de variables psicológicas y situacionales en el apoyo (House, Umberson y Landis, 1988). Así, los resultados obtenidos en la investigación de Gracia y Herrero (2004) mostraron la influen- 
cia en el apoyo social de diversos determinantes personales, incluyendo el estrés percibido, la autoestima y la depresión. Estos resultados son especialmente relevantes, ya que los autores usaron la misma medida de apoyo social utilizada en el presente estudio en el marco de un diseño longitudinal, lo que permite explorar de manera eficaz las relaciones de causalidad entre las variables. Por otro lado, la revisión realizada por Schwarzbach et al. (2014) para el caso específico de las personas mayores sugiere la existencia de un efecto estadísticamente negativo del apoyo social en los niveles de depresión, sobre todo en investigaciones que utilizan medidas funcionales de apoyo. En esta línea, es de especial relevancia el planteamiento realizado por Newcomb (1990), al señalar que la asociación entre apoyo social y salud/enfermedad mental que a menudo es hallada en datos transversales ha de ser interpretada en el marco de una relación recíproca, bidireccional. Dado el diseño transversal utilizado en la investigación descrita en el presente artículo, es preciso atender a consideraciones de este tipo para evitar una interpretación estrecha o unidireccional de la relación entre apoyo social y depresión en el caso de las personas mayores.

\section{Conclusiones}

En un trabajo reciente, Thoits (2011) destaca el análisis de la relación entre fuentes y tipos de apoyo social como uno de los debates de mayor relevancia en dicho ámbito de investigación. La autora defiende la posibilidad de establecer una diferenciación básica entre fuentes primarias (personas significativas) y fuentes secundarias de apoyo (personas similares). En este contexto, y en contraste con las hipótesis de especificidad del apoyo, "la mayoría de los estresores dan lugar a necesidades de apoyo emocional y ayuda en el afrontamiento activo (...). Los tipos de apoyo emocional y ayuda en el

\section{Referencias}

Altintas, E., Gallouj, K. y Guerrien, A. (2012). Social support, depression and self-esteem in older persons: cluster analysis results. Annales Medico-Psychologiques, 170, 256-262.

Alvarado, K.A., Temper, D.I., Bresler, C. y Thomas-Dobson, S. (1995). The relationship of religious variables to death depression and death anxiety. Journal of Clinical Psychology, 51, 202-204.

Antonucci T.C. y Akiyama H. (1987). Social networks in adult life and a preliminary examination of the convoy model. Journal of Gerontology, 42, 519-527.

Antonucci, T.C. (1981). Frontiers in aging. Attachment and social support across the life-span. En A. Lee (Ed.), Frontiers in aging (pp. 43-62). Pullman: Washington State University.

Antonucci, T.C., Fuhrer, R. y Dartigues, J. (1997). Social relations and depressive symptomatology in a sample of community-dwelling French older adults. Psychology and Aging, 12, 189-195.

Antonucci, T.C., Lansford, J.E. y Akiyama, H. (2001). Impact of positive and negative aspects of marital relationships and friendships on wellbeing of older adults. Applied Developmental Science, 5, 68-75.doi: 10.1207/S1532480XADS0502_2

Arechabala, M. C. y Miranda, C. (2002). Validación de una escala de apoyo social percibido en un grupo de adultos mayores adscritos a un programa de hipertensión de la región Metropolitana. Ciencia y Enfermería, 8, 49-55. doi:10.4067/S0717-95532002000100007 afrontamiento que ayudarán a reducir el deterioro psicológico diferirán según la fuente" (Thoits, 2011, p. 159). Los resultados obtenidos en nuestro estudio apoyan parcialmente este planteamiento, ya que la red significativa de apoyo de las personas mayores investigadas cumple funciones tanto instrumentales como emocionales. Ahora bien, al tratar de explicar la depresión nuestro modelo incluye exclusivamente al cónyuge y a los hijos. Pareciera que para la depresión, solo estas fuentes son relevantes. En este punto, es posible que para dar cuenta del efecto del apoyo sobre el bienestar y el deterioro de los tipos y fuentes de apoyo social sea necesario considerar las características concretas del deterioro específico que estemos investigando (por ejemplo, la depresión) (Cutrona, Russel y Rose, 1986).

No todas las relaciones de apoyo desembocan necesariamente en resultados saludables. En este punto, el argumento de Thoits (2011) supone una aportación sobresaliente, ya que el efecto positivo del apoyo social en el bienestar dependería del tipo de apoyo emocional e instrumental que ofreciera la fuente. No todo apoyo emocional y/o instrumental generaría efectos saludables sobre las personas. Eso es, precisamente, lo que sugieren nuestros resultados. En este punto investigaciones de carácter cualitativo (en cierto sentido, una recuperación de la influencia de Brown y Harris, 1978) que se ocupen de la identificación y análisis de los comportamientos (dimensión fenomenológica) de apoyo en función de la fuente de procedencia se presentan como una alternativa con un potencial notable de aportación al conocimiento de los mecanismos que vinculan apoyo social y depresión en el caso de las personas mayores.

Agradecimientos.- Esta investigación ha sido posible gracias a la financiación del programa "Becas Chiles" de CONICYT (Ref. 018/2295).

Baker, F.M. y Espino D.V. (1997) A Spanish version of the Geriatric Depression Scale in Mexican-American elders. International Journal of Geriatric Psychiatry, 12, 21-25.

Barrera, M. (2000). Social support research in Community Psychology. En J. Rappaport y E. Seidman (Eds.), Handbook of Community Psychology (pp. 215-245). New York: Luwer Academic/Plenum Publishers.

Barros, C. y Muñoz, M. (2003). Relaciones e intercambios familiares del adulto mayor. Perspectivas, 12, 23-30.

Barton, Y.A., Miller, L., Wickramaratne, Gameroff, M.J. y Weissman, M.M. (2013). Religious attendance and social adjustment as protective against depression: A 10-year prospective study. Journal of Affective Disorders, 146, 53-57.doi: 10.1016/j.jad.2012.08.037

Beel-Bates, C.A., Ingersoll-Dayton, B. y Nelson, E. (2007). Deference as a form of reciprocity among residents in assisted living. Research on Aging, 29, 626-643.doi: 10.1177/0164027507305925

Brink, T.L., Yesavage, J.A. , Lum. O., Heersema, P.H., Adey, M. y Rose, T.L (1982). Screening tests for geriatric depression. Clinical Gerontology, 1, $37-$ 43.

Brown, G.W. y Harris, T. (1978). Social origins of depression. A study of psychiatric disorder in women. London: Tavistock.

Bukov, A., Maas, I. y Lampert, T. (2002). Social participation in very old age: cross-sectional and longitudinal findings from BASE. Journal of Gerontology, Series B Psychological Sciences and Social Sciences, 57, 510-517. 
Chao, S.F. (2011). Assessing social support and depressive symptoms in older Chinese Adults: a longitudinal perspective. Aging Mental Health, 15(6), 765-774.doi: 10.1080/13607863.2011.562182

Chappell, N.L. y Funk, L.M. (2011). Social support, Caregiving, and Aging. Canadian Journal on Aging, 30, 355-70.doi: 10.1017/S0714980811000316

Choi, N.G. y McDougall, G. (2009). Unmet needs and depressive symptoms among low-income older adults. Journal of Gerontological Social Work, 52, 567-583. doi: 10.1080/01634370802609270

Chou, K.L. y Chi, I. (2003). Reciprocal relationship between social support and depressive symptoms among Chinese elderly. Aging and Mental Health, 7, 224-231.

Cohen, S. y McKay, G. (1984). Social support, stress, and the buffering hypothesis: A theoretical analysis. In A. Baum, S.E. Taylor, y J.E. Singer (Eds.), Handbook of psychology and health. Vol. IV.Social psychological aspects of bealth (253-267). Hillsdale, NJ: Erlbaum.

Cohen, S. y Wills, T.A. (1985). Stress, social support and the buffering hypothesis: A theoretical analysis. Psychological Bulletin, 98, 310-357.

Cruza-Guet, M.C. (2008). A longitudinal analysis of the relationship between social support and psychological distress among Hispanic elders in Miami, Florida. Dissertation Abstracts International Section A: Humanities and Social Sciences. Lehigh University. (MSTAR 869825227; 2011-99070-463).

Cutrona, C., Russell, D. y Rose, J. (1986). Social support and adaptation to stress by the elderly. Psychology and Aging, 1, 47-54.

Dean, A., Kolody, B. y Wood, P. (1990). Effects of social support from various sources on depression in elderly persons. Journal of Health and Social Behavior, 31, 148-161.

Díaz Veiga, P. (1987). Evaluación del apoyo social. En R. FernándezBallesteros (Ed.), El ambiente. Análisis psicológico (pp. 125-149). Madrid: Pirámide.

Feng, D., Ji, L. y Xu, L. (2014). Mediating effect of social support on the association between functional disability and psychological distress in older adults in rural China: Does age make a difference? PLoS ONE, 9(6), e100945.doi: 10.1371/journal.pone.0100945

Fernández-Ballesteros, R. (2000). Gerontología social. Madrid: Pirámide.

Fiori, K.L., Antonucci, T.C. y Cortina K.S. (2006). Social network typologies and mental health among older adults. The Journals of Gerontology Series B: Psychological Sciences and Social Sciences, 61(1), 25-32.

Fowler, K., Wareham-Fowler, S. y Barnes, C. (2013). Social context and depression severity and duration in Canadian men and women: exploring the influence of social support and sense of community belongingness. Journal of Applied Social Psychology, 43, E85-E96.doi: 10.1111/jasp.12050

George, L.K., Kinghorn, W.A., Koenig, H.G., Gammon, P. y Blazer, D.G. (2013). Why gerontologists should care about empirical research on religion and health: Transdisciplinary perspectives. The Gerontologist, 53, 898-906.doi: 10.1093/geront/gnt002

González-Celis, A.L. y Gómez Benito, J. (2013). Spirituality and quality of life and its effect on depression in older adults in Mexico. Pychology, 4, 178-182.doi: $10.4236 /$ psych.2013.43027

Gracia, E. y Herrero, J. (2004). Personal and situational determinants of relationship-specific perceptions of social support. Social Behavior and Personality, 32, 459-476.

Gracia, E. y Herrero, J. (2006). La comunidad como fuente de apoyo social: Evaluación e implicaciones en los ámbitos individual y comunitario. Revista Latinoamericana de Psicología, 38, 327-342.

Gracia, E. y Musitu, G. (1990). Integración y participación en la comunidad: una conceptualización empirica del apoyo social comunitario. Psicología Comunitaria. Valencia: NAU Llibres.

Gracia, E., Herrero, J. y Musitu, G. (2002). Evaluación de recursos y estresores psicosociales en la comunidad. Madrid: Síntesis.

Harpham, T., Grant, E. y Thomas, E. (2002). Measuring social capital within health surveys: Key issues. Health Policy and Planning, 17, 106-111.doi: 10.1093/heapol/17.1.106

Herrera, M.S. y Kornfeld, R. (2008). Relaciones familiares y bienestar de los adultos mayores en Chile. Expansiva, 131, 1-15.

Herrera, M.S., Barros, C. y Fernández, M.B. (2011). Predictors of Quality of Life in Old Age: A Multivariate Study in Chile. Journal of Population Ageing, 4, 121-139.doi: 10.1007/s12062-011-9043-7

Herrera, M.S., Rojas, M., Campos, F. y Fernández, B. (2013). Encuesta Nacional de calidad de vida en la vejez 2013. Servicio Nacional de Adultos Mayores: Santiago de Chile.
Herrero, J. y Gracia, E. (2007). Measuring perceived community support: Factorial structure, longitudinal invariance, and predictive validity of the PCSQ (Perceived Community Support Questionnaire). Journal of Com munity Psychology, 35, 197-217.doi: 10.1002/jcop.20143

House, J.S., Umberson, D. y Landis, K.R. (1988). Structures and processes of social support. Annual Review of Sociology, 14, 293-318.

Hoyl, T., Valenzuela, E. y Marín, P.P. (2000). Depresión en el adulto mayor: evaluación preliminar de la efectividad, como instrumento de tamizaje, de la versión de 5 items de la Escala de depresión Geriátrica. Revista Médica de Chile, 128, 1199-1204.

Kahn, R.L. y Antonucci, T.C. (1984). Social supports of the elderly: Family, friends, professionals (Publication No. AG01632). Bethesda, MD: National Institute of Aging.

Kline, R.B. (2005). Principles and practice of structural equation modeling. Nueva York: Guilford.

von dem Knesebeck, O., Dragano, N., Moebus, S., Jöckel, K.H., Erbel, R. y Siegrist, J. (2009). Stressful experiences in social relationships and ill health. Psychotherapie, Psychosomatik, medizinische Psychologie, 59(5): 186-193. doi: 10.1055/s-2008-1067421

Koizumiy, Y., Awata, S., Kuriyama, S., Ohmori, K., Hozawa, A., Seki, T., ... Tsuji, I. (2005). Association between social support and depression status in the elderly: Results of a 1-year community-based prospective cohort study in Japan. Psychiatry and Clinical Neurosciences, 59, 563-569.doi: 10.1111/j.1440-1819.2005.01415.x

Larsson, K. y Silverstein, M. (2004). The effects of marital and parental status on informal support and service utilization: A study of older Swedes living alone. Journal of Aging Studies, 18, 231-244.doi: 10.1016/j.jaging.2004.01.001

Li, H., Morrow-Howell, N., Proctor, E. y Rubin, E. (2013). Social support resources and post-acute recovery for older adults with major depression. Community Mental Health Journal, 49 (4), 419-426.doi: 10.1007/s10597-012-9567-1

Liang, J., Krause, N.M. y Bennett, J.M. (2001). Social exchange and wellbeing: Is giving better than receiving? Psychology and Aging, 16, 511-523.

Lin, N. (1986). Conceptualizing social support. En N. Lin, A. Dean y W. Ensel (Eds.). Social support, life events, and depression (pp. 17-30). Nueva York: Academic Press.

Litwin, H. (2011). The association between social network relationships and depressive symptoms among older Americans: what matters most? International Psychogeriatrics, 23(6), 930-940. doi: 10.1017/S1041610211000251

Luppa, M., Luck, T., Köning, H.H., Angermeyer, M.C. y Riedel-Heller, S.G. (2012). Natural course of depressive symptoms in late life. An 8-year population-based prospective study. Journal of Affective Disorders, 142, 166-171.doi: 10.1016/j.jad.2012.05.009

Mackean, R. y Abbott-Chapman, J. (2012). Older people's perceived health and wellbeing: The contribution of peer-run community-based organizations. Health Sociology Review, 21, 47-57.

Mair, C., Diez-Roux, A.V. y Morenoff, J.D. (2010). Neighborhood stressors and social support as predictors of depressive symptoms in the Chicago Community Adult Health Study. Health \& Place, 16, 811-819.doi: 10.1016/j.healthplace.2010.04.006

Marcil-Gratton, N. y Légaré, J. (1992). Will reduced fertility lead to greater isolation in old age for tomorrow's elderly? Canadian Journal on Aging, 11 54-71.

Mardia, K.V. (1970). Measures of multivariate skewness and kurtosis with applications. Biometrika, 57, 519-530.

McLaughlin, D., Leung, J., Pachana, N., Flicker, L., Hankey, G. y Dobson, A. (2012). Social support and subsequent disability: it is not the size of your network that counts. Age and Ageing, 41 (5), 674-677.doi: 10.1093/ageing/afs036

Moos, R.H., Brennan, P.L., Schutte, K.K. y Moos, B.S. (2010). Social and financial resources and high-risk alcohol consumption among older adults. Alcoholism: Clinical and Experimental Research, 34, 646-654. doi: 10.1111/j.1530-0277.2009.01133.x

von Mühlenbrock, F., Gómez, R., González, M., Rojas, A., Vargas, L. y von Mühlenbrock, C. (2011). Prevalencia de Depresión en pacientes mayores de 60 años hospitalizados en el Servicio de Medicina Interna del Hospital Militar de Santiago. Revista chilena de neuropsiquiatría, 4, 331-337. doi: 10.4067/S0717-92272011000400004 
Murrell, S.A., Norris, F.H. y Chipley, Q.T. (1992). Functional versus structural social support, desirable events, and positive affect in older adults. Psychology and Aging, 7, 562-570.

Newcomb, M.D. (1990). Social support and personal characteristics: a developmental and interactional perspective. Journal of Social and Clinical Psychology, 9, 54-68.

Okabayashi, H., Liang, J., Krause, N., Akiyama, H. y Sugisawa, H. (2004). Mental health among older adults in Japan: Do sources of social support and negative interaction make a difference? Social Science y Medicine, 59, 2259-2270.doi: 10.1016/j.socscimed.2004.02.024

Park, N.S., Jang, Y.T., Lee, B.S., Ko, J.E. y Chiriboga, D.A. (2014). The impact of social resources on depressive symptoms in racially and ethnically diverse older adults: Variations by groups with differing health risks. Research on Aging, 36, 322-342.doi: 10.1177/0164027513486991

Peters, G.R., Hoyt, D.R., Babchuk, N., Kaiser, M. y Iijima,Y. (1987). Primary-group support systems of the aged. Research on Aging, 9, 392-416.

Quinn, W.H., Hughston, G.A. y Hunter, D.J. (1984). Preservation of independence through nonformal support systems: Implications and promise. En W.H. Quinn y G.A. Hughston (Eds.), Independent aging: family and social systems perspectives (pp. 215-237). Tumbridge Wells: Aspen Systems Corporation.

Robitaille, A., Orpana, H. y McIntosh, C.N. (2012). Reciprocal relationship between social support and psychological distress among a national sample of older adults: An autoregressive cross-lagged model. Canadian Journal on Aging, 31, 13-24.doi: 10.1017/S0714980811000560

Roth, S., Lee, K.H. y Yoon, D.P. (2013). General well-being of Korean immigrant elders: The significance of religiousness/spirituality and social support. Journal of Social Service Research, 39, 483-497.doi: 10.1080/01488376.2012.709451

Rushing, N.C., Corsentino, E., Hames, J.L., Sach-Ericsson, N. y Steffens, D.C. (2013). The relationship of religious involvement indicators and social support to current and past suicidality among depressed older adults. Aging \& Mental Health, 17, 366-374.doi: 10.1080/13607863.2012.738414
Sánchez, E. y Barrón, A. (2003). Social psychology of mental health. The social structure and personality perspective. The Spanish Journal of Psychology, 6, 311.doi: $10.1017 / \mathrm{S} 1138741600005163$

Schwarzbach, M., Luppa, M., Forstmeier, S., König, H. y Riedel-Heller, S.G. (2014). Social relations and depression in late life - a systematic review. International Journal of Psychiatry, 29, 1-21.doi: 10.1002/gps.3971

Shaw, B.A., Krause, N., Liang, J. y Bennett, J. (2007). Tracking changes in social relations throughout late life. The Journals of Gerontology. Series B: Psychological Sciencies and Social Sciences, 62, 90-99.

Sternthal, M.J., Williams, D.R., Musick, M.A. y Buck, A.C. (2010). Depression, anxiety, and religious life: A search for mediators. Journal of Health and Social Behavior, 51 (3), 343- 359. doi: 10.1177/0022146510378237

Thoits, P.A. (1983). Multiple identities and psychological well-being: A reformulation and test of the social isolation hypothesis. American Sociological Review, 48, 174-187.

Thoits, P.A. (1995). Stress, coping, and social support processes: Where are we? What are next?. Journal of Health and Social Behaviour, Extra Issue, 5379.

Thoits, P.A. (2011). Mechanisms linking social ties and support to physical and mental health. Journal of Health y Social Behavior, 52, 145-161. doi: $10.1177 / 0022146510395592$

Vanderhorst, R.K. y McLaren, S. (2005). Social relationships as predictors of depression and suicidal ideation in older adults. Aging and Mental Health, 9, 517-525.

Vera, P., Buela, G. y Zych, I. (2010). Chilean experimental version of the state- trait depression questionnaire (ST-DEP): State subscale (S-DEP). Psychological Reports, 1, 65-77.

Yoon, D.P. (2008). Factors affecting subjective well-being for rural elderly individuals the importance of spirituality, religiousness, and social support. Journal of Religion and Spirituality in Social Work, 25, 59-75. doi: 10.1300/J377v25n02_04

(Artículo recibido: 15-04-2013; revisado: 10-06-2014; aceptado: 28-08-2014) 